\title{
DIAGNOSIS OF DUCHENNE AND BECKER MUSCULAR DYSTROPHIES BY DNA POLYMORPHISM
}

\author{
Yoshiko Akita, ${ }^{1}$ Shigeo OHNo, ${ }^{1}$ Jun Goto, ${ }^{2}$ \\ Imaharu NAKANO, ${ }^{2}$ Masami TAKATSU, ${ }^{3}$ \\ Hideo Sugrta, ${ }^{4}$ and Koichi SuzukI ${ }^{1}$ \\ ${ }^{1}$ Department of Molecular Biology, The Tokyo Metropolitan Institute \\ of Medical Science, Bunkyo-ku, Tokyo 113, Japan \\ ${ }^{2}$ Department of Neurology, Shimoshizu National Hospital \\ and Sanatorium, Yotsukaido, Chiba 284, Japan \\ ${ }^{3}$ Division of Neurology, Department of Internal Medicine, Teikyo \\ University School of Medicine, Itabashi-ku, Tokyo 173, Japan \\ ${ }^{4}$ Division of Neuromuscular Research, National Institute of \\ Neuroscience, NCNP, Kodaira, Tokyo 187, Japan
}

Summary Twenty families affected with Duchenne (DMD) or Becker (BMD) muscular dystrophy from the Japanese population were investigated using six DNA polymorphisms at the pERT87 locus. Allele frequencies for these polymorphisms were different from those found in Caucasians and $94 \%$ of unrelated women were informative for linkage analysis. DMD and BMD phenotypes cosegregated with a particular allele in each family with no obligate recombinants among 50 meiotic events. Among 20 unrelated affected males, one boy had a deletion of at least 52 kilobases (kb) surrounding the pERT87 region.

\section{INTRODUCTION}

Duchenne muscular dystrophy (DMD) is one of the most common and serious $\mathrm{X}$-linked recessive disorders with a frequency of about 1 in 3,000-5,000 newborn males (Gardner-Medwin, 1980). Becker muscular dystrophy (BMD) is clinically similar to DMD in the pattern of muscle involvement, but follows a more benign course. The underlying basic defect in these diseases and an effective treatment are unknown. Until recently, prenatal diagnosis has not been feasible and carrier detection in families at risk for DMD or BMD has depended on a somewhat equivocal test for serum creatine kinase (CK) activity (Sugita and Tyler, 1963; Harper, 1982).

Received February 18, 1987; Accepted March 23, 1987 
DNA restriction fragment length polymorphisms (RFLPs) have recently been used for diagnosis of various diseases including DMD. Kunkel and coworkers (1985) have isolated DNA fragments, pERT87 (DXS164), from the Xp21 region deleted in a DMD male patient. They successfully used subcloned fragments (pERT87-1, pERT87-8, and pERT87-15) from the pERT87 locus as probes to detect RFLPs diagnostic of DMD (Monaco et al., 1985; Kunkel et al., 1986). However, the applicability of these subclones for DMD diagnosis in populations other than American and European Caucasian populations has not yet been evaluated. Moreover, the usefulness of these probes in BMD prediction has not been fully elucidated.

In this study, we examined the feasibility of these three pERT87 subclones to detect DMD and BMD patients and carriers in affected Japanese families. The results indicate that these probes are highly useful for analyses of Japanese DMD and BMD families.

\section{MATERIALS AND METHODS}

Subjects. Unrelated healthy volunteers and members of twelve families with Duchenne or Becker muscular dystrophy from the Japanese population were investigated for DNA polymorphisms.

The diagnosis of DMD or BMD was made on the basis of clinical findings and progression of the disease, serum CK levels, muscle histology and electromyography were examined by one of the authors (J.G., I.N., M.T., or H.S.). Pedigrees with more than two affected males in one or more generations or in relatives were examined to exclude isolated cases possibly arising by new mutation.

Restriction endonuclease analysis. Genomic DNA was prepared from lymphocytes in 10-20 ml of heparinized peripheral blood by the guanidium/cesium chloride method (Maniatis et al., 1982). Each DNA sample $(5-10 \mu \mathrm{g})$ was digested to completion with 2-fold excess units of the appropriate restriction endonuclease (Xmnl, TaqI, Bst NI, or BstXI, New England Biolabs and Takara Shuzo) in the buffer recommended by the manufacturer. The DNA samples (1.5 $\mu \mathrm{g}$ each) were separated by electrophoresis on $0.7 \%$ agarose gels and transferred to nitrocellulose filters by the method of Southern (1975). Inserts of subclones pERT87-1, pERT87-8 and pERT87-15 (provided by L. Kunkel) were labeled with [ $\left.{ }^{32} \mathrm{P}\right] \mathrm{dCTP}$ (New England Nuclear) using an Amersham nick translation kit. Hybridization of nitrocellulose filters was undertaken as described by Maniatis et al. (1982). After overnight incubation at $65^{\circ} \mathrm{C}$, the excess probe was washed off in $2 \times \mathrm{SSC}(1 \times \mathrm{SSC}=\mathrm{NaCl} 0.15$ $\mathrm{mol} / \mathrm{liter}$, trisodium citrate $0.015 \mathrm{~mol} / \mathrm{liter}), 0.1 \%$ sodium dodecyl sulfate (SDS) at room temperature for $30 \mathrm{~min}$, followed by a wash at $55^{\circ} \mathrm{C}$ in $0.1 \times \mathrm{SSC}, 0.1 \% \mathrm{SDS}$ for $50 \mathrm{~min}$.

Human genomic DNA segments (OTC-d, -e, and $-f$ ) in the locus for ornithine transcarbamylase (OTC), which maps to Xp21 proximal to the DMD locus (Lindgren et al., 1984; Davies et al., 1985), were isolated from a human genomic library 
(provided by T. Maniatis) using rat OTC complimentary DNA (Takiguchi et al., 1984) as a probe. Two genomic DNA fragments (1-1 and 1-4) were isolated from a human $\mathrm{X}$ chromosome library (provided by Y. Sakaki). These segments were demonstrated to be unique fragments of the $X$ chromosome by dosage analysis using a panel of DNAs prepared from normal male lymphocytes (46XY) and the lymphoblastoid cell line GM 1416, 48XXXX (Human Genetic Mutant Cell Repository, Camden, NJ).

\section{RESULTS}

\section{Frequency of polymorphisms}

The allele frequency and heterozygosity of three probes of the pERT87 (DXS164) locus in Japanese individuals including healthy volunteers and unrelated obligate carriers of DMD or BMD are shown in Table 1, together with the results from American and European Caucasian groups (Kunkel et al., 1986). Racial differences were observed in the frequencies of several polymorphic sites, e.g. the alleles for pERT87-8 with both Bst XI $\left(\chi^{2}=15.39\right.$, d.f. $\left.=1, \mathrm{p}<0.001\right)$ and TaqI $\left(\chi^{2}=13.31\right.$, d.fo $=$ $1, \mathrm{p}<0.001)$ and for pERT87-15 with TaqI $\left(\chi^{2}=4.98\right.$, d.f. $\left.=1, \mathrm{p}<0.05\right)$. Although allele frequencies differed considerably between the two populations, the expected heterozygosities at all loci except the TaqI site for pERT87-15 were approximately $40-50 \%$ in the Japanese population, similar to those found in Americans and Europeans. The expected heterozygosities corresponded well to the observed heterozygosities, that is, the proportion of heterozygous females relative to the total females examined. These data imply that, in the Japanese population, each of three pERT87 subclones is highly informative for identifying females at risk of being DMD carriers.

Of particular interest is the proportion of DMD families in which DNA diagnoses can be made by a combination of these three pERT87 probes and four restriction enzymes. The proportion of females heterozygous at one or two of the polymorphic Bst NI and $X m n I$ sites detected by pERT $87-1$ was 14 out of $25(56 \%)$, although the heterozygosity calculated from the allele frequencies at the two polymorphic sites was $75 \%$ (Table 2). Using the BstXI site for pERT87-8 combined with the TaqI site provided little additional information ( 7 out of $18,39 \%$ ) for detecting DMD by linkage analysis. In contrast, using the TaqI site for pERT8715 in combination with the $X m n I$ site improved the test applicability from $50 \%$ to $68 \%$ (13 out of 19 ), although the heterozygosity at the TaqI site was somewhat low $(24 \%)$. Finally, when the two enzyme-defined sites of the pERT87-15 probe were used in combination with the polymorphic sites of pERT87-1 and pERT87-8, 30 out of the 32 unrelated Japanese women tested $(94 \%)$ were informative for linkage analysis.

\section{Nonrandom association of polymorphic sites}

The above data suggest that there is a strong linkage disequilibrium between 
Table 1. Allele frequency and heterozygosity of DNA polymorphisms for pERT87.

\begin{tabular}{|c|c|c|c|c|c|c|}
\hline \multirow{2}{*}{ Probe } & \multirow{2}{*}{$\begin{array}{l}\text { Restriction } \\
\text { enzyme }\end{array}$} & \multicolumn{3}{|c|}{ Allele frequency } & \multicolumn{2}{|c|}{ Heterozygosity $(\%)$} \\
\hline & & \multicolumn{2}{|c|}{$\begin{array}{c}\text { Americali \& } \\
\text { European }^{a}\end{array}$} & Japanese & $\begin{array}{c}\text { American \& } \\
\text { European }\end{array}$ & Japanese \\
\hline & & & & & \multicolumn{2}{|c|}{ Expected (Observed) } \\
\hline \multirow[t]{4}{*}{ pERT87-1 } & $X m n I$ & $A:$ & 0.66 & 0.53 & 45 & $50(53)$ \\
\hline & & $a:$ & 0.34 & $\begin{array}{c}0.47 \\
(\mathrm{n}=70)\end{array}$ & & \\
\hline & Bst NI & $C:$ & 0.63 & 0.48 & 47 & $50\langle 54\rangle$ \\
\hline & & $c:$ & 0.37 & $\begin{array}{c}0.52 \\
(n=54)\end{array}$ & & \\
\hline \multirow[t]{4}{*}{ pERT87-8 } & Bst XI & $D:$ & 0.60 & $0.26^{b}$ & 48 & 38 (39) \\
\hline & & $d:$ & 0.40 & $\begin{array}{c}0.74 \\
(n=58)\end{array}$ & & \\
\hline & $\operatorname{Taq} \mathbf{I}$ & $E:$ & 0.71 & $0.38^{\mathrm{c}}$ & 41 & $47(40)$ \\
\hline & & $e:$ & 0.29 & $\begin{array}{c}0.62 \\
(n==52)\end{array}$ & & \\
\hline \multirow[t]{4}{*}{ pERT87-15 } & TaqI & $F:$ & 0.67 & $0.85 \mathrm{~d}$ & 44 & $26(24)$ \\
\hline & & $f:$ & 0.33 & $\begin{array}{c}0.15 \\
(n=52)\end{array}$ & & \\
\hline & $X m n \Gamma$ & $B:$ & 0.68 & 0.59 & 44 & $48(50)$ \\
\hline & & $b:$ & 0.32 & $\begin{array}{c}0.41 \\
(n=70)\end{array}$ & & \\
\hline
\end{tabular}

a Allele frequencies in American and European populations are cited from the data by Kunkel et al. (1986). Expected heterozygosities in both Japanese and Caucasian groups were calculated from the allele frequencies at each site. Observed heterozygosity represents the proportion of heterozygous females relative to total females examined. $\mathrm{n}$, number of observed chromosomes. $\mathrm{b} \chi_{1}{ }^{2}=15.39$, $\mathrm{p}<0.001 ;{ }^{\mathrm{c}} \chi_{1}{ }^{2}=13.32, \mathrm{p}<0.001 ; \mathrm{d} \chi_{1}{ }^{2}=4.98, \mathrm{p}<0.05$.

Table 2. Applicability of polymorphic sites for the pERT87 subclones in DMD Diagnosis.

\begin{tabular}{|c|c|c|c|}
\hline \multirow{2}{*}{ Probe } & \multirow{2}{*}{ Restriction site } & \multicolumn{2}{|c|}{ Applicability $(\%)^{a}$} \\
\hline & & Observed & Expected \\
\hline pERT87-1 & $X m n \mathrm{I}$ and $B s t \mathrm{NI}$ & $56^{b}(14 / 25)$ & 75 \\
\hline pERT87-8 & Bst XI and TaqI & $39 \mathrm{c}(7 / 18)$ & 67 \\
\hline pERT87-15 & $X m n I$ and $T a q I$ & $68(13 / 19)$ & 62 \\
\hline pERT87-1, .15 & $X m n I$ & $81 \quad(26 / 32)$ & 74 \\
\hline All & $X m n \mathbf{I}, T a q \mathrm{l}, B s t \mathrm{XI}$, and/or Bst $\mathrm{NI}$ & $94(30 / 32)$ & - \\
\hline
\end{tabular}

a Applicability represents the percentage of women who are informative for one or more loci. Values in parentheses are the number of informative women/total women. Expected applicability is calculated from the observed allele frequencies at each site. $\mathrm{b} \chi_{1}{ }^{2}=4.81, \mathrm{p}<0.05 ;$ c $\chi_{\mathrm{L}}{ }^{2}=6.38 . \mathrm{p}<0.025$. 
Table 3. Nonrandom association of the $X m a I$ and $B s t$ NI polymorphic sites detected by pERT87-1 in Japanese population.

\begin{tabular}{lccc}
\hline \multirow{2}{*}{ Genotype } & $\begin{array}{c}\text { Number } \\
\text { observed }\end{array}$ & \multicolumn{2}{c}{ Frequency } \\
\cline { 3 - 4 } & 5 & 0.20 & Observed \\
ac/ac & 6 & 0.24 & 0.06 \\
$A C / a c, A c / a C$ & 12 & 0.48 & 0.06 \\
$A c / a c$ & 1 & 0.04 & 0.25 \\
$A c / A C$ & 1 & 0.04 & 0.13 \\
Others & 0 & 0 & 0.14 \\
\hline
\end{tabular}

Deruced haplotype: Common; ar $(49 \%)$ and $A C(45 \%)$

Rare, Ac and $a C$

Haplotype frequency was deduced from the frequency of each homozygote. $A$ and $a$, alleles for $X m m I$ polymorphism; $C$ and $c$, alleles for Bst NI polymorphism. $\chi_{3}{ }^{2}=38.96, p<0.001$.

the two polymorphic sites detected by pERT87-1 or by pERT87-8, whereas the two polymorphic sites for pERT87-15 are closer to equilibrium with each other. For studying linkage disequilibrium, we deduced the haplotypes from the genotypes for the two polymorphisms for pERT87-1. In theory, 10 possible genotypes (though $A C / a c$ can not be distinguished from $A C / a C$ ) exist as a result of random combination of four haplotypes, $A C, A c, a C$, and $a c$. However, only four out of the 10 theoretical genotypes (ac/ac; $A C / a c$ and $A c / a C ; A C / A C$ ) accounted for $92 \%$ of Japanese individuals (Table 3 ). The population frequencies for these genotypes were 0.24 , 0.48 and 0.20 , while their expected frequencies based on the observed allele frequencies at the two sites were $0.06,0.25$, and 0.06 , respectively. The frequency of haplotype $A C$ (p) or ac (q) calculated from the frequency of each homozygote is $\mathrm{p}=0.45$ or $\mathrm{q}=0.49$, respectively. The estimated frequency of genotype $A C$ ac is $2 \mathrm{pq}=0.44$, similar to the sum $(0.48)$ of genotypes $A C / a C$ and $A C / a C$. Thus, genom type $A c / a C$ is probably rare. The frequencies for $A c / a c$ and $A c / A C$ are also very low. These data suggest that two haplotypes, $A C$ and $a c$, are common and the others are rare in the Japanese population. The striking linkage between the $X \mathrm{~mm} \mathrm{I}$ and $B s t$ NI sites (allele $A$ and $C$ or allele $a$ and $c$ ) for pERT87 1 probably reduces their usefulness for the diagnosis of DMD. The fact that the two XmnI sites detected by pERT87-1 and pERT87-15 increased the applicability of prediction from $50 \%$ to $81 \%$ (26 of 32 tested females) suggests that the pERT87-15 region is randomly associated with the pERT87-1 region (Table 2). Further analysis of three pERT87 markers in pairwise combinations indicates that they are closer to equilibrium with one another (data not showri).

Linkage studies in DMD and BMD families

Of 16 DMD and BMD families from the Japanese population studied, DNA 
diagnosis of 15 was possible by the three probes. The segregation of RFLPs demonstrated Mendelian inheritance with no obligate recombinants in 50 meiotic events in 14 DMD and 1BMD families. Representative data are shown in Figs. 1, 2 and 4. In family $J$ (Fig. 1), since the affected boy received from his mother a $8.8 \mathrm{~kb}$ $X m n I$ fragment (allele $A$ ) hybridizing with pERT87-1 while another son with a 7.5 $\mathrm{kb}$ band (allele $a$ ) is normal, it is presumed that the maternal $\mathrm{X}$ chromosome carrying allele $A$ is responsible for DMD. A daughter who has received allele $A$ from her mother has been diagnosed as being a carrier of DMD, a prediction that is consistent with her increased CK level. Prenatal diagnosis of her male fetus will be possible by examining for the presence of 1.6 and $1.2 \mathrm{~kb} \mathrm{XmnI} \mathrm{fragments} \mathrm{(allele} B$ ) hybridizing with pERT87-15 which also cosegregate with DMD. In family $P$ the two affected boys have received allele $B$ from their grandmother via their mothers, whereas the daughter has received a $2.8 \mathrm{~kb}$ fragment (allele $b$ ) from her mother and 1.6 and $1.2 \mathrm{~kb}$ fragments from her father. Thus, she has been diagnosed as normal with a probable accuracy of $95 \%$. Likewise the daughter in family $\mathrm{E}$ is probably normal because she does not carry the DMD-X chromosome cosegregating with the $2.2 \mathrm{~kb}$ band (allele $d$ ). In families $\mathrm{H}$ and $\mathrm{C}$, DMD cosegregates with a $2.8 \mathrm{~kb}$ $X m n I$ fragment (allele $b$ ) for pERT87-15 and a $3.3 \mathrm{~kb}$ TaqI fragment (allele $f$ ) for pERT87-15, respectively.

In family GK, the mother and her affected son were previously shown to have an interstitial deletion in band Xp21 by cytogenetic analysis (Saito et al., 1986). Segregation of two XmnI RFLPs for pERT87-1 and pERT87-15 in this pedigree shows that the DMD boy bears the deletion in the pERT87 locus and suggests that his mother is hemizygous for this locus (Fig. 2). To characterize this point more precisely, we performed a dosage analysis. The hybridization intensity of the mother in the pERT87 locus detected by two probes (pERT87-1 and pERT87-15) was similar to that seen for the single $X$ chromosome in control males rather than that of the two $X$ chromosomes in control females (Fig. 3). This confirms that she is heterozygous for the deletion found in her DMD son. The two pERT87 subclones span a region of $52 \mathrm{~kb}$ of the genomic sequence (Kunkel et al., 1986), and, therefore, the lower limit of this deletion is $52 \mathrm{~kb}$. However, the ornithine transcarbamylase locus (OTC-d, -e, and -f) and two other DNA segments (1-1 and 1-4) of the X chromosome were present in the DNA of the DMD boy and his mother. This is consistent with previous cytological studies. Except for this case, none of the 18 unrelated DMD and two BMD males had a deletion of the pERT87 region.

Figure 4 shows a BMD family segregating for the pERT87-8 and pERT87-15

Fig. 1. DMD families segregating for the pERT87 polymorphisms. Southern blot analysis of DNA from family members was performed as described in MATERIALs and MerHods. Sizes of the hybridizable fragments (alfeles) are indicated in kilobase (kb). In family $\mathbf{J}$, bands $A$, a and $B$, b were detected by pERT87-15 and pERT87. 1 , respectively. Symbols are as follows: $\square$, normal male; $O$, normal female; $\mathbf{n}$, affected male; 0 , obligate carrier; 0 , manifesting carrier as detected by this experiment. 


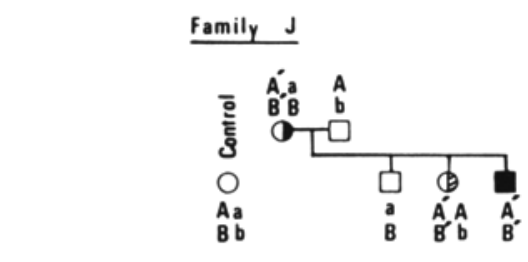

Family $P$

allele $\mathrm{kb}$

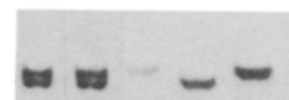

A $\quad 8.8=\square=$

b $2.8-\ldots=$

B $\sum_{1.2-}^{1.6-}=-$

PERT $(87-1)+(87-15)$

$X m n$ I

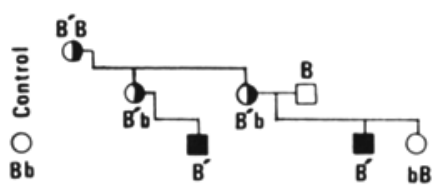

allele $\mathrm{kb}$

b $2.8-$

$B<\left\langle_{1.2-}^{1.6-}\right.$

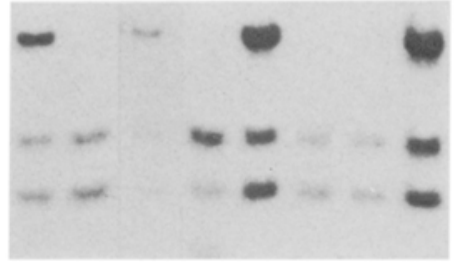

PERT 87-15

Family E

$X_{m n}$ I

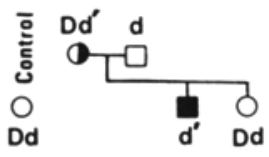

allele kb

0 4.4-

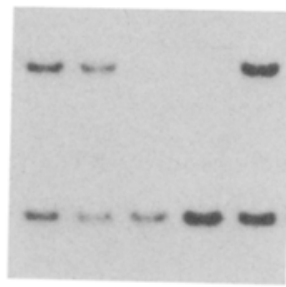

Family H

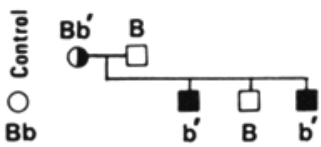

allele $\mathrm{kb}$

b $2.8-$

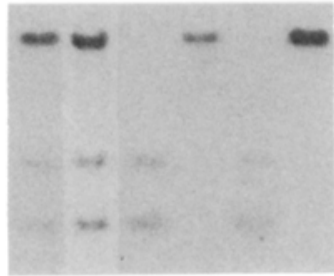

PERT $87-15$

$X \mathrm{mn} \mathbf{I}$
PERT 87-8

BstX I

Family C

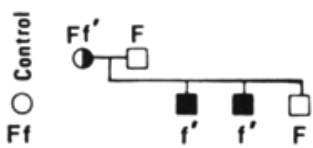

allele kb

f $\quad 3.3= \pm=$
PERT 87-15

Taq I

Fig. 1 . 


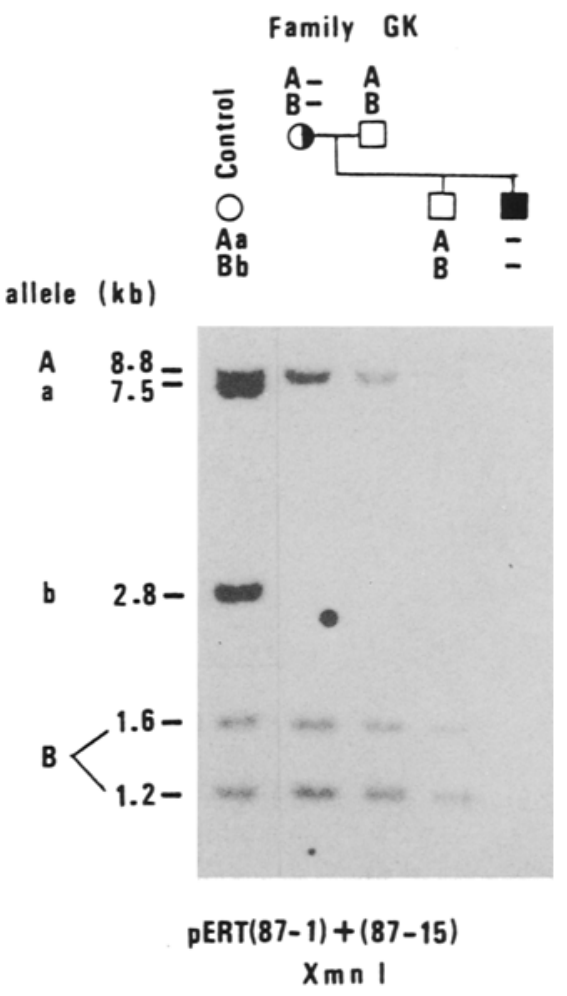

Fig. 2. Analysis of deletions for pERT87-1 and pERT87-15 in a DMD family. Symbols are the same as in Fig. 1.

polymorphisms with no obligate recombinants in eight meiotic events. In this pedigree, BMD is carried by the $\mathrm{X}$ chromosome with a $3.8 \mathrm{~kb}$ TaqI fragment (allele e) detected by pERT $87-8$ in the two mothers who are sisters. Therefore, three relative females who have received allele $e$ from each mother are probably carriers of BMD. The linkage analysis in this pedigree indicates that the BMD gene is located near the pERT87 locus in the same region as the DMD gene.

\section{DISCUSSION}

The general applicability of DNA diagnosis requires both highly polymorphic DNA markers and a closely linked relationship between the disease in question and the marker loci. The close linkage between the DMD locus and the pERT87 probes has been previously reported (Monaco et al., 1985; Kunkel et al., 1986) and is confirmed by this study. In this report, we have investigated the applicability of these three DNA fragments to the diagnosis of DMD or BMD, especially in the Japanese population, because the proportion of heterozygotes often varies with race (Chak- 


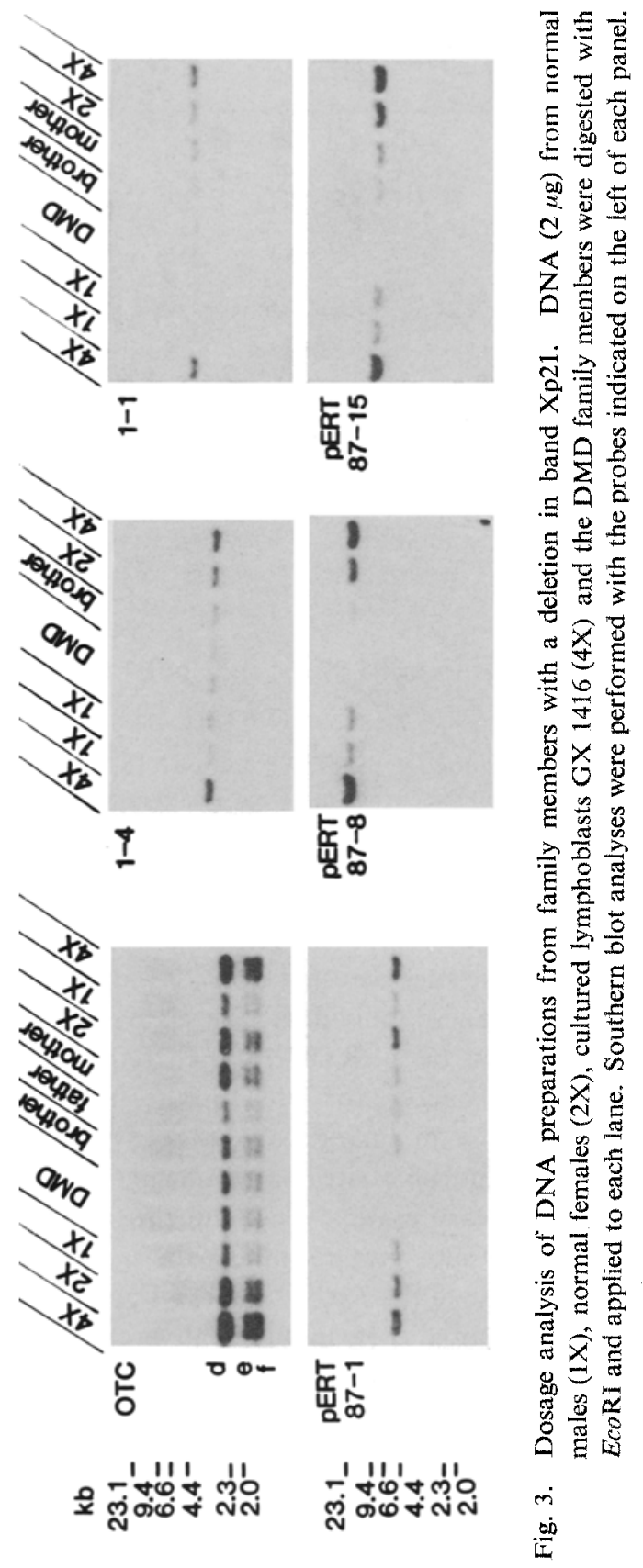




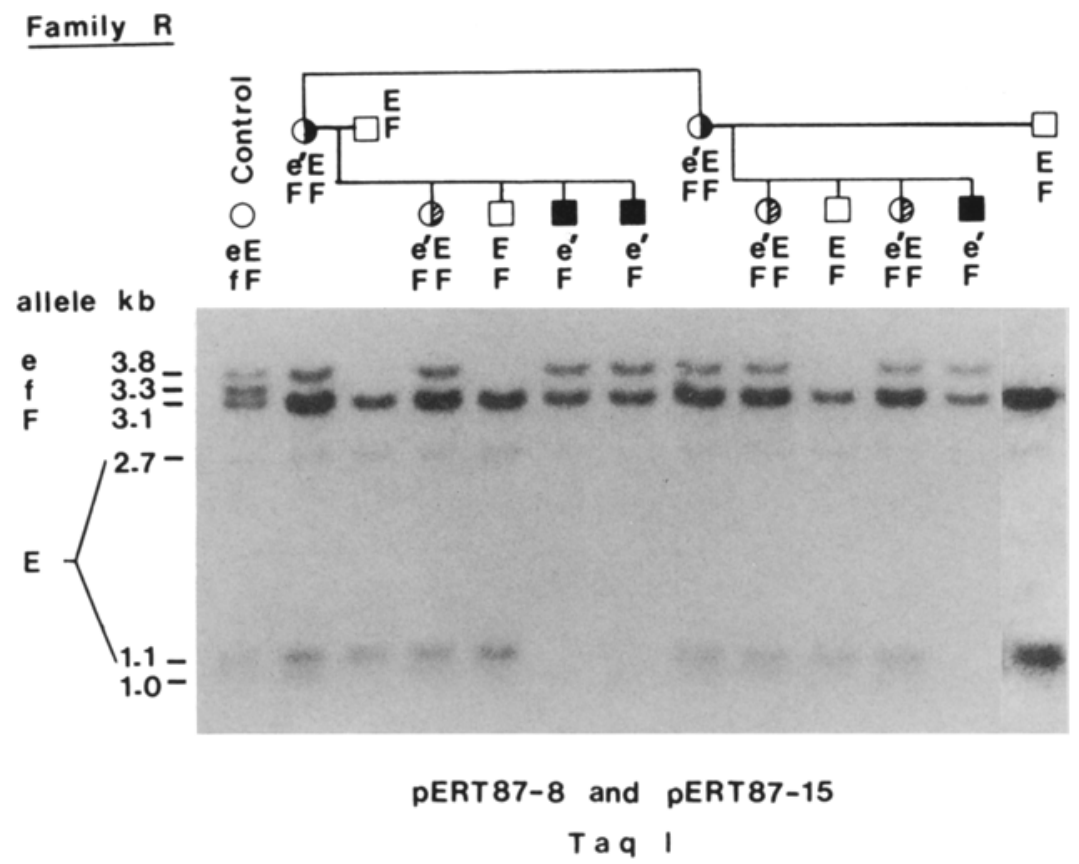

Fig. 4. BMD family segregating for pERT87-8 and pERT87-15 polymorphisms. Southern blot analysis of DNA from maternal relatives affected with BMD is shown below the pedigree. Alleles $E, e$ and a $1.0 \mathrm{~kb}$ invariant band were detected by pERT87-8, and allele $F$ and $f$ were detected by pERT87-15.

ravarti et al., 1984: Elbein et al., 1986). Although, a racial difference in the frequencies of three polymorphic sites detected by Bst XI and TaqI in the pERT87 locus (Table 1) was observed, Japanese individuals are highly polymorphic for all RFLPs except the TaqI site detected by pERT87-15. Therefore, RFLPs in the pERT87 locus are good polymorphic markers for DMD in the Japanese population.

To obtain high efficiency in linkage analysis, several markers are necessary. However, some DNA polymorphic sites show strong linkage disequilibria (Antonarakis et al., 1982; Chakravarti et al., 1986), reducing their usefulness. Therefore, two or more markers sometimes give essentially the same results. Such a relationship probably exists in the case of the Bst NI and XmnI polymorphic sites for pERT871 (Table 3), since only one of the 12 uninformative cases examined by the XmnI RFLP for pERT87-1 was informative by the Bst NI RFLP. Since the RFLPs for pERT87-15 and those for pERT87-1 and pERT87-8 showed random associations, a set of $X m n I$ RFLPs detected by both pERT87-1 and pERT87-15 is the most informative and provides high efficiency with one operation within the same panel. In order to minimize the number of tests per case necessary to obtain information for DNA diagnosis, we recommend performing the screening in the following order: screening with (1) the two $X m n I$ sites for pERT87-1 and pERT87-15, (2) the two 
TaqI sites for pERT87-8 and pERT87-15, and/or (3) the BstXI site for pERT87-8.

The accuracy of diagnosis is inversely related to recombination between the disease and marker loci. In this study, we found no recombination with the six RFLPs seen by three pERT87 subclones in Japanese individuals. However, it has been reported that the pERT87 locus apparently recombines with a frequency of $5-6 \%$ in American and European populations (Fischbeck et al., 1986: Kunkel et al., 1986). Therefore, for a more precise diagnosis of DMD, pERT 87 probes should be used in combination with other Xp21 flanking markers.

The DMD patient with a deletion in band Xp21 (family GK) is suffering from complex glycerol kinase deficiency (GKD), a syndrome characterized by congenital adrenal hypoplasia (CAH) and mental retardation, but his ornithine transcarbamylase activity is normal. Our Southern blot analysis confirmed that the OTC locus was present in this DMD patient. Francke (1984) reported a female with an Xp21 deletion who was diagnosed as being a carrier of OTC deficiency and CGD (chronic granulomatous disease). Recently, it has been shown that the X-linked CGD locus is proximal to the DMD locus and lies within the Xp21 region (Baehner et al., 1986). Since the case in this report is not suffering from CGD, we speculate that one break point in this deletion is distal to the CGD and OTC loci, and the other point is distal to the DMD locus. Precise analysis of the end points of this deletion will help to determine the position of the GK locus relative to the CGD and DMD loci.

The present results together with those reported previously (Brown et al., 1985; Wilcox et al., 1985: Kunkel et al., 1986) demonstrate a close linkage of BMD with pERT87 subclones, and suggest that BMD and DMD are caused by different mutations of the same locus or else that their loci, while distinct, are located very close to each other.

Acknowledgments We thank Dr. L.M. Kunkel (Childrens' Hospital, Boston, Mass.), Dr. M. Mori (Kumamoto University, Kumamoto, Japan), Dr. T. Maniatis (Harvard University, Cambridge, Mass.) and Dr. Y. Sakaki (Kyushu University, Fukuoka, Japan) for providing the pERT87(DXS164) probes, rat OTC cDNA clone, a human genomic DNA library, and a human X chromosome library, respectively; Mr. Y. Konno for technical assistance. This study was supported in part by Grantsin-Aid for Scientific Research from the Ministry of Education, Science and Culture, and grants from the National Center of Neurology and Psychiatry (NCNP) of the Ministry of Health and Welfare of Japan.

\section{REFERENCES}

Antonarakis, S.E., Boehm, C.D., Giardina, P.J.V., and Kazazian, H.H., Jr. 1982. Nonrandom association of polymorphic restriction sites in the $\beta$-globin gene cluster. Proc. Natl. Acad. Sci. USA 79: 137-141.

Baehner, R.L., Kunkel, L.M., Monaco, A.P., Haines, J.L., Conneally, P.M., Palmer, C., Heerema, N., and Orkin, S.H. 1986. DNA linkage analysis of X chromosome-linked chronic granum lomatous disease. Proc. Natl. Akad. Sci. USA 83: 3398-3401. 
Brown, C.S., Thomas, N.S.T., Sarfarazi, M., Davies, K.E., Kunkel, L., Pearson, P.L., Kingston, H.M., Shaw, D.J., and Harper, P.S. 1985. Genetic linkage relationships of seven DNA probes with Duchenne and Becker muscular dystrophy. Hum. Genet. 71: 62-74.

Chakravarti, A., Phillips, J.A., III, Mellits, K.H., Buetow, K.H., and Seeburg, P.H. 1984. Patterns of polymorphism and linkage disequilibrium suggest independent origins of the human growth hormone gene cluster. Proc. Natl. Acad. Sci. USA 81: 6085-6089.

Chakravarti, A., Elbein, S.C., and Permutt, M.A. 1986. Evidence for increased recombination near the human insulin gene: Implication for disease association studies. Proc. Natl. Acad. Sci. USA 83: 1045-1049.

Davies, K.E., Speer, A., Herrmann, F., Spiegler, A.W.J., McGlade, S., Hofker, M.H., Briand, P., Hanke, R., Schwartz, M., Steinbicker, V., Szibor, R., Korner, H., Sommer, D., Pearson, P.L., and Coutelle, Ch. 1985. Human X chromosome markers and Duchenne muscular dystrophy. Nucleic Acids Res. 13: 3419-3426.

Elbein, S.C., Corsetti, L., Ullrich, A., and Permutt, M.A. 1986. Multiple restriction fragment length polymorphisms at the insulin receptor locus: A highly informative marker for linkage analysis. Proc. Natl. Acad. Sci. USA 83: 5223-5227.

Fischbeck, K.H., Ritter, A.W., Tirschwell, D.L., Kunkel, L.M., Bertelson, C.J., Monaco, A.P., Hejtmancik, J.F., Boehm, C., Ionasescu, V., Ionasescu, R., Pericak-Vance, M., Kandt, R., and Roses, A.D. 1986. Recombination with pERT87 (DXS164) in families with X-linked muscular dystrophy. Lancet ii: 104.

Francke, U. 1984. Random X inactivation resulting in mosaic nullisomy of region Xp21.1-p21.3 associated with heterozygosity for ornithine transcarbamylase deficiency and for chronic granulomatous disease. Cytogenet. Cell Genet. 38: 298-307.

Gardner-Medwin, D. 1980. Clinical features and classification of the muscular dystrophies. $B r$. Med. Bull. 36: 109-115.

Harper, P.S. 1982. Carrier detection in Duchenne muscular dystrophy: a critical assessment. In Disorders of the Motor Unit, Schotland, D.L., ed., Wiley, New York, pp. 821-844.

Kunkel, L.M., Monaco, A.P., Middlesworth, W., Ochs, H.D., and Latt, S.A. 1985. Specific cloning of DNA fragments absent from the DNA of a male patient with an X chromosome deletion. Proc. Natl. Acad. Sci. USA 82: 4778-4782.

Kunkel, L.M. and co-authors. 1986. Analysis of deletions in DNA from patients with Becker and Duchenne muscular dystrophy. Nature 322: 73-77.

Lindgren, V., de Martinville, B., Horwich, A.L., Rosenberg, L.E., and Francke, U. 1984. Human ornithine transcarbamylase locus mapped to band Xp21.1 near the Duchenne muscular dystrophy locus. Science 226: 698-700.

Maniatis, T., Fritsch, E.F., and Sambrook, J. 1982. Molecular Cloning, Cold Spring Harber Laboratory, New York.

Monaco, A.P., Bertelson, C.J., Middlesworth, W., Colletti, C.-A., Aldridge, J., Fischbeck, K.H., Bartlett, R., Pericak-Vance, M.A., Roses, A.D., and Kunkel, L.M. 1985. Detection of deletions spanning the Duchenne muscular dystrophy locus using a tightly linked DNA segment. Nature 316: 842-845.

Saito, F., Goto, J., Kakinuma, H., Nakamura, F., Murayama, S., Nakano, I., and Tonomura, A. 1986. Inherited Xp2l deletion in a boy with complex glycerol kinase deficiency syndrome. Clin. Genet. 29: 92-93.

Southern, E.M. 1975. Detection of specific sequences among DNA fragments separated by gel electrophoresis. J. Mol. Biol. 98: 503-517.

Sugita, H. and Tyler, F.H. 1963. Pathogenesis of muscular dystrophy. Trans. Assoc. Am. Physiol. Ixxvi: 231-243.

Takiguchi, M., Miura, S., Mori, M., Tatibana, M., Nagata, S., and Kaziro, Y. 1984. Molecular cloning and nucleotide sequence of cDNA for rat ornithine carbamoyltransferase precursor. Proc. Natl. Acad. Sci. USA 81: 7412-7416.

Wilcox, D.E., Affara, N.A., Yates, J.R.W., Ferguson-Smith, M.A., and Pearson, P.L. 1985. Multipoint linkage analysis of the short arm of the human $X$ chromosome in families with X-linked muscular dystrophy. Hum. Genet. 70:365-375. 